\title{
E-Assessments in der Hochschullehre: Chancen und Herausforderungen
}

\author{
Xenia Valeska Jeremias *, Birgit Sellmer, Ulrike Tippe
}

\section{Zusammenfassung}

Dieser Beitrag stellt die im Rahmen des Forschungsprojekts „SOS - Strukturierung und Optimierung des Selbststudiums" entwickelten Ansätze zur qualitätsgesicherten Erstellung von E-Assessments vor. Er beleuchtet die Anforderungen an E-Assessments unter verschiedenen Aspekten, wobei der Schwerpunkt auf den Möglichkeiten der inhaltlichen Umsetzung von Fragen liegt.

Der erste Teil behandelt die Erstellung von qualitätsgesicherten elektronischen Fragen, indem fünf verbreitete Vorurteile zu E-Assessments beschrieben und widerlegt werden. Beispiele aus dem im Rahmen des oben genannten Projektes erstellten „Leitfaden zur Erstellung von elektronischen Fragen und Tests der TH Wildau [FH]“ illustrieren dies. Im zweiten Teil werden praktische Erfahrungen mit dem Einsatz von E-Assessments in der Lehre vorgestellt. Dies erfolgt insbesondere unter den Aspekten Einarbeitungsaufwand seitens der Lehrenden, der benötigten personellen Ressourcen sowie der technischen und organisatorischen Rahmenbedingungen.

\section{Abstract}

This article presents an approach for quality assurance of e-assessments that was developed by the research project „SOS - Strukturierung und Optimierung des Selbststudiums" ("Structuring and Improving Self-Studies"). Different requirements for e-assessments are highlighted, emphasising possible implementations in both form and content.

The first part deals with the development of electronic questions and focuses on quality demands. Five prevalent misconceptions relating to e-assessments are discussed and refuted. Examples gathered from the „TH Wildau Guidelines for Development of Electronic Questions and Tests", which were addressed by the above mentioned research project, illustrate the line of reasoning. The second part covers practical experience using e-assessments in teaching at a tertiary institution. In so doing, the focal points address the efforts required by academics to familiarise themselves with the software, the necessary human resources as well as the general technical and organisational conditions to facilitate this.

\section{EINLEITUNG UND ZIELSETZUNG}

Seit 2011 fördert das Bundesministerium für Bildung und Forschung im Rahmen des Qualitätspakts Lehre die Verbesserung der Lehre an Hochschulen. Die TH Wildau [FH] gehört mit ihrem Projekt „SOS - Strukturierung und Optimierung des Selbststudiums "1 zu den geförderten Hochschulen und legt dabei mit Mathematik und Rechnungswesen einen Schwerpunkt auf zwei Fachgebiete, die über die Studiengänge hinweg für viele Studierende eine Herausforderung darstellen.
Des Weiteren werden übergreifende Maßnahmen zur Erhöhung der Lehrqualität entwickelt. Hierzu gehören Blended-Learning-Angebote, für die strukturierte Online-Materialien erstellt werden, sowie die Konzeptionierung und Erprobung von hochschulweiten Unterstützungsstrukturen für das Selbststudium. In diesem Rahmen wird u. a. - ausgehend von den Bedarfen der Lehrenden und Studierenden - seit Mitte 2012 der Einsatz von E-Assessments erprobt.

In diesem Beitrag werden die Ansätze des Projekts SOS zur Sicherung quali- tativ hochwertiger elektronischer Fragen und Tests in den wissenschaftlichen Kontext eingeordnet. Im zweiten Teil des Beitrags werden Erfahrungen mit dem Einsatz elektronischer Tests in der Lehre diskutiert.

\section{CHARAKTERISTIKA VON E-ASSESSMENTS}

Mit dem Begriff E-Assessment werden alle elektronischen Formen von Prüfungen, (Selbst-)Tests und Bewertungen gefasst, also nicht nur prüfungsrechtlich relevante Prüfungen.

${ }^{1}$ Erreichbar unter http://th-wildau.de/selbststudium. Das Projekt wird aus Mitteln des Bundesministeriums für Bildung und Forschung unter dem Förderkennzeichen 01PL11042 im Rahmen des Qualitätspakts Lehre gefördert. 
E-Assessments bestehen aus verschiedenen Elementen und können diagnostisch, formativ oder summativ eingesetzt werden (Handke \& Schäfer 2012: $150 \mathrm{ff}$.).

Diagnostische E-Assessments: Hierbei handelt es sich typischerweise um Orientierungstests für Studieninteressierte, Einstufungstests für Teilnehmer/innen von Vorbereitungskursen und Eingangstests für Studienanfänger/innen, um beispielsweise die sinnvolle Einteilung in Lerngruppen oder zielgerichtete weitere Lernangebote zu unterstützen.

Formative E-Assessments: Diese können studienbegleitend eingesetzt werden, um Studierenden wie auch Lehrenden eine Rückmeldung über den aktuellen Lernstand zu geben.

Summative E-Assessments: Die Modulprüfung am Ende des Semesters ist ein typisches Beispiel für ein summatives Assessment, das die Leistung von Studierenden bzw. Prüfungsteilnehmenden abschließend prüft.

Im Hochschulbereich ist seit einigen Jahren ein zunehmender Bedarf an Standardisierung und Automatisierung von Prüfungen zu verzeichnen, sodass E-Assessments mittlerweile aus der Diskussion über E-Learning- bzw. Blended-Learning-Szenarien nicht wegzudenken sind. Steht doch vielfach die Hoffnung dahinter, dass durch automatisierte Auswertung und/oder durch Mehrfachverwendung der Fragen (Aufbau eines Fragenpools) die Korrektur und auch die Zusammenstellung von Klausuren insbesondere bei großen Seminargruppen deutlich erleichtert werden. Ob diese Hoffnungen berechtigt sind, sei an dieser Stelle zunächst einmal dahingestellt. Festzuhalten ist vielmehr, dass derartige Szenarien die Hochschulen vor neue Herausforderungen stellen, die sich über die drei Bereiche

- Technik (sowohl Hard- als auch Software),

- Organisation (u.a. Raumausstattung und -größe, Login-Daten, Datenschutz und -sicherheit, Archivierung) sowie
- Inhalt (u. a. Fragetypen und -niveau)

erstrecken (Biella et al. 2010: 3 ff.).

Dieser Artikel fokussiert schwerpunktmäßig den letzten Aspekt, den Inhalt und die Möglichkeiten zur Erstellung von niveauvollen Fragen, und beschäftigt sich $u$. a. mit der Frage, ob E-Assessments so angelegt werden können, dass diese vom Anspruch und der Art der Fragen mit "traditionellen“ Prüfungsformen mithalten können. Des Weiteren wird auf den Aufwand zur Erstellung und die Vor- und Nachteile der Nutzung von E-Assessments eingegangen.

Aus Sicht der Autorinnen rechtfertigt sich ein möglicher zusätzlicher Aufwand bei der Erstellung und Durchführung von E-Assessments, wenn

- die dort gestellten Fragen so abgebildet werden können, dass sie sich vom Niveau und Anspruch her für Prüfungen im Hochschulbereich eignen und

- diese zusätzlich so weit wie möglich automatisiert ausgewertet werden können.

Im nächsten Abschnitt werden zu beiden Aspekten verschiedene Behauptungen aufgestellt, die sich mit teilweise existierenden Vorurteilen zu E-Assessments auseinandersetzen und diese widerlegen.

\section{E-ASSESSMENTS: FÜNF VORURTEILE}

\section{Vorurteil 1: Elektronische Tests enthalten ausschließlich Multiple- Choice-Fragen.}

Tatsächlich sind viele elektronische Tests überwiegend aus MultipleChoice-Fragen (MC-Fragen) zusammengesetzt; dies muss jedoch nicht so sein. Testtools bieten eine Reihe von weiteren Fragetypen an, die ebenfalls automatisch ausgewertet werden können, beispielsweise numerische Fragen, bei denen eine Zahl als Antwort gesucht ist. Auch Lückentext-, Zuordnungs- oder Rangfolgeaufgaben sind in der Regel automatisch auswertbar. Ob sich die
Frage als Prüfungsfrage eignet, hängt ohnehin nicht allein vom Fragetyp, sondern von der prinzipiellen Gestaltung der Frage ab. Empfehlenswert ist eine Mischung der Frageformate, da abwechslungsreiche Tests für die Nutzer/innen attraktiver sind.

Vorurteil 2: Mit Multiple-ChoiceFragen kann nur Faktenwissen abgefragt werden.

Kompetenzen, die in Studienmodulen erworben werden sollen, erfordern in der Regel mindestens die Fähigkeit, Gelerntes adäquat auf neue Sachverhalte anzuwenden, zum Teil auch eine anspruchsvollere Auseinandersetzung mit dem Lerngegenstand, beispielsweise durch Vergleiche oder Bewertungen verschiedener Methoden. Die reine Wiedergabe von deklarativem Wissen, d. h. von Fakten, ist demnach selten geeignet für Aufgaben und Prüfungen im Hochschulbereich. Der Schwerpunkt liegt vielmehr auf Fragestellungen, die prozedurales oder konditionales Wissen verlangen. Während deklaratives Wissen lediglich das Wissen um die Existenz bestimmter Strategien und Tatsachen beinhaltet, umfasst prozedurales Wissen darüber hinaus das Wissen um die Anwendung dieser Strategien; konditionales Wissen beinhaltet zudem das Wissen, wann bzw. in welchem Kontext welche Strategie sinnvoll eingesetzt werden kann (Paris et al. 1983: 302 ff.). In der Tat sind Fragetypen wie numerische Fragen häufig besser dazu geeignet, prozedurales und konditionales Wissen zu überprüfen als MC-Fragen; jedoch ist dies (siehe Abbildung 1) auch mit MC-Fragen möglich, wenn diese entsprechend konstruiert werden (Bücking 2011: 40).

Abbildung 1 zeigt drei Varianten einer mathematischen Aufgabenstellung. Die erste Variante in Form einer MCFrage ist insofern problematisch, als sie ausschließlich Faktenwissen abfragt. Die zweite Variante - ebenfalls eine MC-Frage - wäre zu bevorzugen, da in dieser Form Anwendungswissen geprüft wird. Allerdings ist hier eine Annäherung an die richtige Lösung über das Ausschlussprinzip möglich. Noch besser ist jedoch die 
dritte Variante, eine numerische Frage, die den Lösungsweg nur minimal vorgibt und einer „konventionellen“ Frage in Papierform am nächsten kommt. Mit dieser Gestaltung der Frage wird prozedurales Wissen geprüft.

Die Aufgabengestaltung unter Qualitätssicherungsaspekten ist insbesondere bei MC-Fragen nicht ganz trivial. Daher wurde an der TH Wildau [FH] ein Leitfaden ${ }^{2}$ für die Erstellung von Aufgaben entwickelt. Zu den Qualitätsaspekten gehört u.a., dass die Antwortvorgaben dicht an der richtigen Antwort liegen, etwa gleich lang und gleich komplex sind, sich jedoch nicht überschneiden sowie möglichst typische Fehler und Fehlkonzepte enthalten. Selbstverständlich sollte es nicht möglich sein, die Frage ohne Fachkenntnis, d.h. allein durch die Kombination der in der Frage und den Antwortmöglichkeiten enthaltenen Informationen, richtig zu beantworten (Krebs 2004: 6 ff.). Wird dies bei der Aufgabengestaltung beachtet und der Fokus auf prozedurales Wissen gelegt, wie beispielhaft in Abbildung 2 gezeigt, spricht aber nichts gegen die Verwendung von MC-Aufgaben. Somit wird deutlich, dass die Möglichkeiten solcher Fragen nur in geringem Maße ausgeschöpft werden, wenn diese ausschließlich Fakten (z. B. Begriffsdefinitionen) abfragen.

In den folgenden Beispielen kann die richtige Antwort jeweils nur gegeben werden, wenn die entsprechenden Zusammenhänge grundsätzlich verstanden wurden. Die Studierenden müssen das entsprechende Wissen also anwenden und nicht nur reproduzieren. Des Weiteren zeigt das erste Beispiel eine Möglichkeit, wie eine Aufgabe, bei der auf Papier ein Graph gezeichnet werden müsste, durch das Verwenden von Bildern als elektronische Variante gestaltet werden kann. Im zweiten Beispiel geht es im Gegensatz zur reinen Abfrage von bestimmten Eigenschaften - um eine Begründung bzw. Erklärung.
Mit welcher Formel können Sie bei einem rechtwinkligen Dreieck die Hypotenuse c berechnen, wenn die beiden Katheten a und b gegeben sind?

$$
\begin{aligned}
& a^{2}+b^{2}=c^{2} \\
& a^{2} \times b^{2}=c^{2} \\
& a+b=c \\
& a^{2}-b^{2}=c^{2}
\end{aligned}
$$

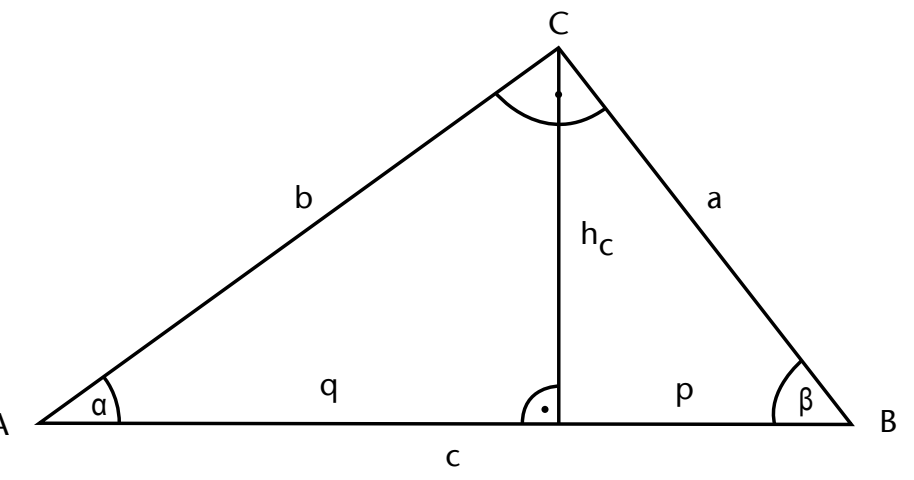

In dem gegebenen Dreieck seien die Katheten $6,3 \mathrm{~cm}$ und $3,6 \mathrm{~cm}$ lang.

Wie lang ist die Hypotenuse?
$5,17 \mathrm{~cm}$
$7,26 \mathrm{~cm}$
$9,90 \mathrm{~cm}$
$22,68 \mathrm{~cm}$

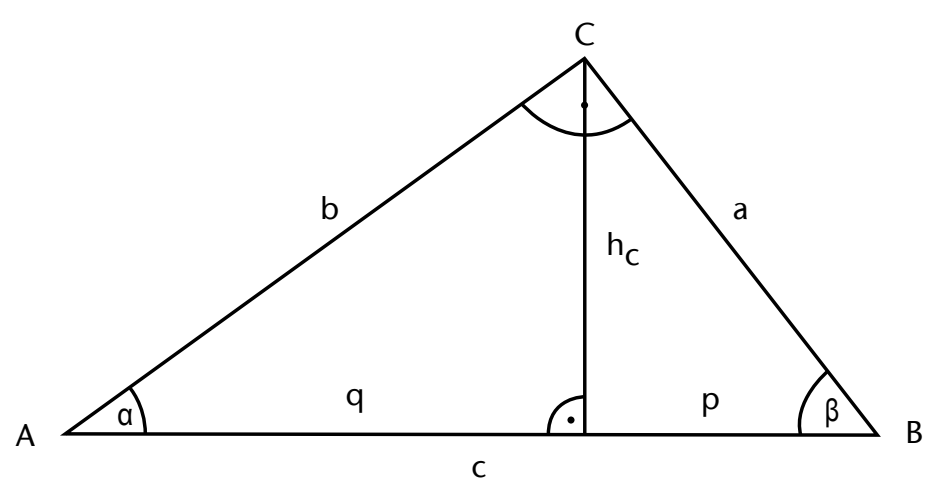

In dem gegebenen Dreieck seien die Katheten $6,3 \mathrm{~cm}$ und $3,6 \mathrm{~cm}$ lang. Wie lang ist die Hypotenuse?

Runden Sie - wenn nötig - auf zwei Stellen nach dem Komma! $c=$ $\mathrm{cm}$

${ }^{2}$ Abrufbar unter www.th-wildau.de/eassessment. Alle hier gezeigten Beispiele sind diesem Leitfaden entnommen. 
Gegeben sei die Funktion $f(x)=9 x-3$

Welcher Graph bildet diese Funktion ab?
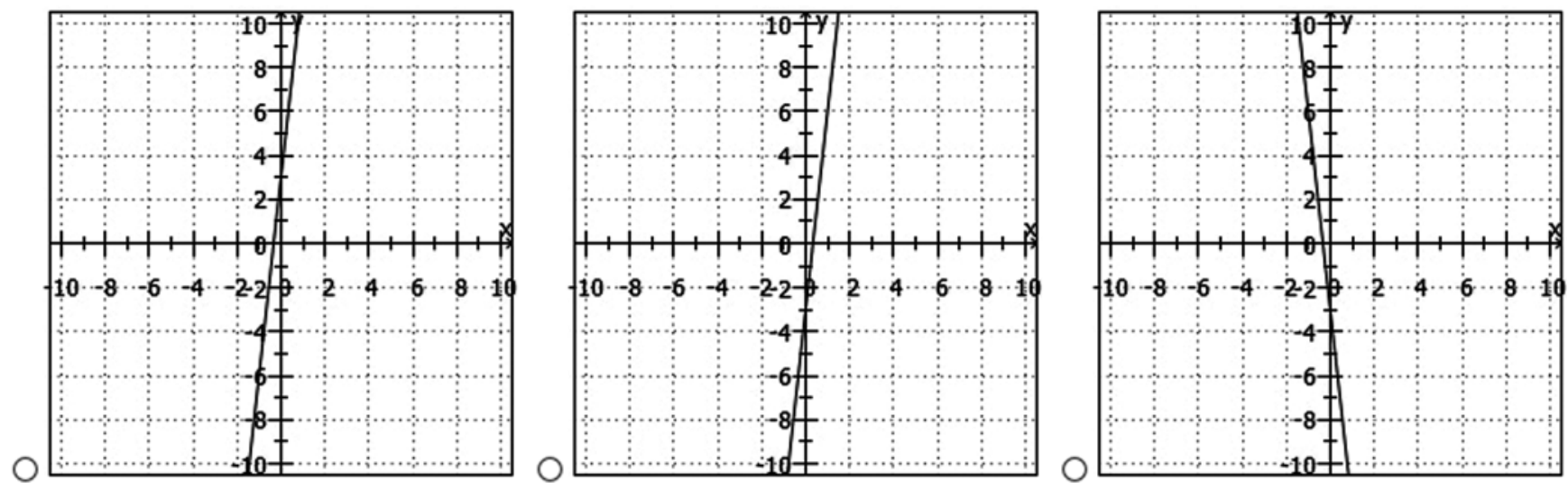

Vorgehensweisen beurteilen / begründen / erklären

Warum hat Wasser einen so hohen Siedepunkt, eine hohe Wärmekapazität und eine hohe Verdampfungswärme?

durch die starke $\mathrm{H}-\mathrm{O}$-sigma-Bindung

durch Dipol-Dipol-Wechselwirkungen

durch die hohe Dichte des Wassers

durch die freien Elektronenpaare am Sauerstoff, die eine Pi-Überlappung eingehen

durch Wasserstoffbrückenbindungen

Abb. 2) Abfrage von prozeduralem Wissen mittels MC-Fragen.

Vorurteil 3: Aufgaben mit längeren Textantworten eignen sich nicht für elektronische Tests.

Sicherlich können Freitextaufgaben nicht automatisch ausgewertet werden, sondern müssen vom Lehrenden kontrolliert und individuell bewertet werden, doch können auch bei diesem Fragetyp Korrekturzeiten eingespart werden. Im Vergleich zu automatisch auswertbaren Fragetypen wie Auswahl-, Zuordnungs- und Rankingfragen werden bei Freitextantworten Korrekturzeiten sogar in höherem Maße eingespart (Schulz \& Apostolopoulos 2011: 37 ff.), was vor allem auf die bessere Lesbarkeit und Struktur der Antworten zurückgeführt wird. Freitextaufgaben können also nicht nur einfach erstellt und in E-Assessments integriert werden - sie sind auch empfehlenswert!

Vorurteil 4: Die Qualitätsansprüche, die an schriftliche Aufgaben gestellt werden, können durch elektronische Tests nicht erfüllt werden.

Die Qualität von Prüfungs- und Übungsaufgaben ist ein integraler Bestandteil guter Lehre. Prüfungsleistungen sind die Grundlage für die Vergabe von Leistungspunkten nach ECTS und somit für den Studienabschluss, der bescheinigt, dass die in den Modulbeschreibungen festgelegten Kenntnisse, Fertigkeiten und Kompetenzen erreicht worden sind. Durch die Prüfungsleistungen - unabhängig von einer schriftlichen oder elektronischen Form - müssen die Studierenden nachweisen, dass sie die entsprechenden Lernziele erreicht haben.

\section{Für jede Art von Test muss gelten:}

- Inhaltliche und formale Richtigkeit

- Validität

- Beachten der rechtlichen Vorgaben
Darüber hinaus muss bei elektronischen Prüfungen gegeben sein:

- Datensicherheit und Datenschutz

- Technische Sicherheit

Bezüglich anderer wichtiger Aspekte der Fragen- und Testerstellung gelten für elektronische Prüfungen im Prinzip die gleichen Anforderungen wie für herkömmliche (Bücking 2011: 40). So muss u. a. eine Kohärenz zwischen Lernziel, Lehr-/Lernform und Prüfungsform bestehen und ein didaktisch sinnvoller Aufbau der Prüfungsfragen gewährleistet sein. Die zu beachtenden qualitätssichernden Aspekte werden bei E-Assessments durch die explizit zu treffenden Entscheidungen bezüglich der Fragengestaltung und -bewertung in mindestens gleicher Weise nahegelegt wie bei „Paper-and-Pencil-Prüfungen“. So verlangt $z$. B. eine Prüfungssoftware in der Regel die vorherige Festlegung 


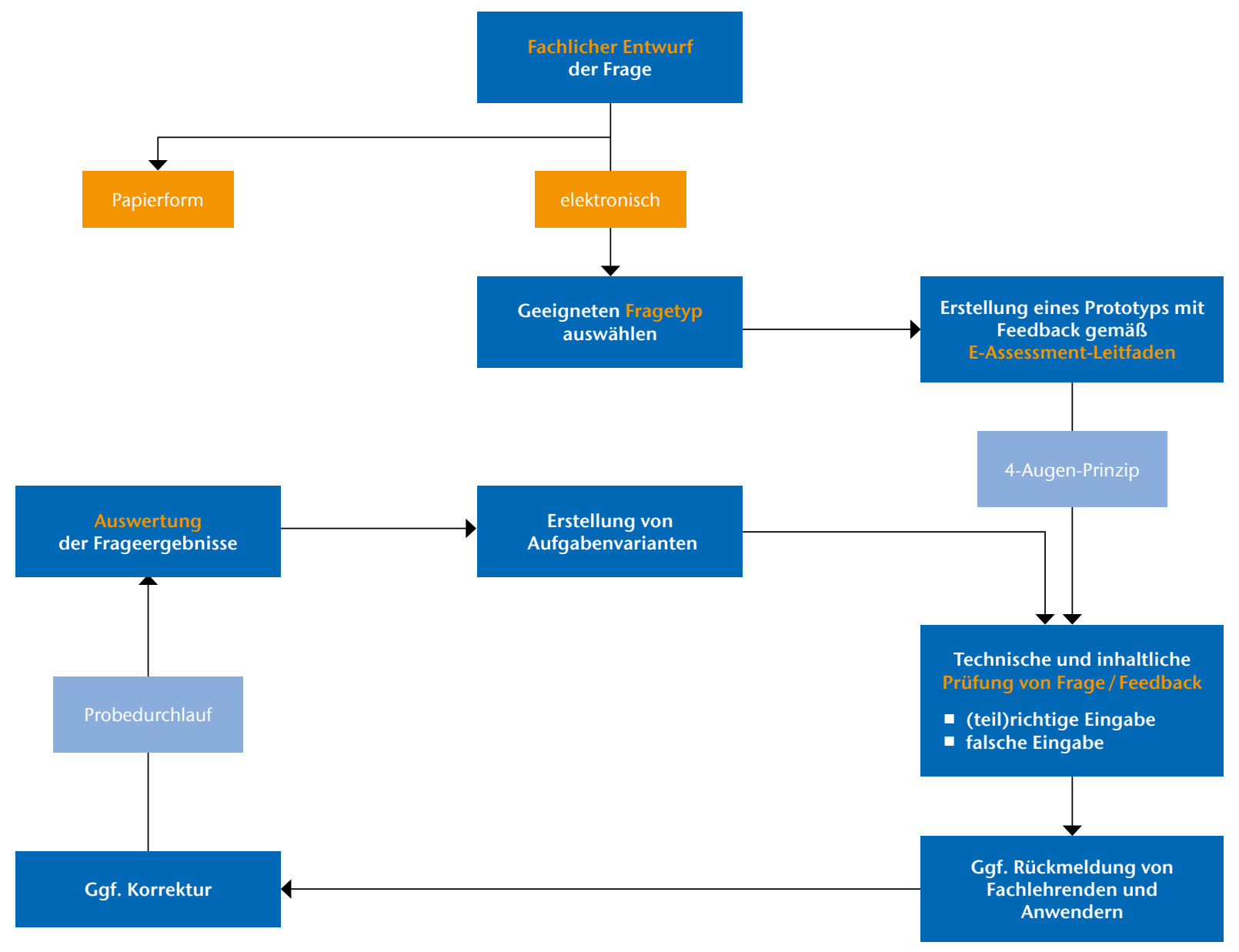

Abb. 3) Ablauf zur Qualitätssicherung von elektronischen Fragen.

der Punkteverteilung. Da für derartige Software ohnehin eine Schulung nötig ist, ist hier ein Rahmen gegeben, in dem Qualitätsaspekte besprochen werden können.

Elektronische Aufgaben müssen in jedem Fall in Übereinstimmung mit den Lernzielen des Moduls erstellt werden (Case \& Swanson 2002: 10). Sie unterscheiden sich dabei in einigen Punkten, je nachdem, ob es sich um Prüfungs- oder Übungsaufgaben handelt. Während bei Prüfungsaufgaben die zu erreichenden Kenntnisse, Fertigkeiten und Kompetenzen auf Modulniveau abgefragt werden, geht es bei Übungsaufgaben vor allem darum, das Verständnis - eventuell durch einen schrittweise ansteigenden Schwierigkeitsgrad - zu fördern. Daher kommt einem differenzierten, lernunterstützend formulierten Feedback bei Übungsaufgaben eine hohe Bedeutung zu. Bei Prüfungsaufgaben stehen hingegen die eindeutig richtige Lösung und die technisch korrekt funktionierende Bewertung im Vordergrund, um die Rechtssicherheit der Prüfung zu gewährleisten. Formale oder technische Fehler haben zwar bei Übungsaufgaben keine derartigen Folgen, wirken aber demotivierend auf die Übenden, sodass sie das Bearbeiten unter Umständen abbrechen. Durch Qualitätssicherungsschleifen sollten daher sowohl inhaltliche als auch technische Fehler für alle elektronischen Aufgaben möglichst ausgeschlossen werden (Ehlers et al. 2013: 235).

Aus Abbildung 3 wird ersichtlich, dass bei der Entwicklung von elektronischen Aufgaben der Gestaltung eines guten Aufgabenprototyps eine hohe Bedeutung zukommt, wobei das Vorliegen eines Leitfadens den Entwicklungsprozess deutlich erleichtert. Aufgabenvarianten, die natürlich ebenfalls auf technische und inhaltliche Korrektheit geprüft werden müssen, können ausgehend von diesem Prototyp mit weniger Aufwand erstellt werden.
Vorurteil 5: Elektronische Selbsttests können nicht sinnvoll in eine Lehrveranstaltung des Typs Vorlesung - Übung integriert werden.

Vielfach ist die Erfahrung, dass Studierende vermeintlich nicht prüfungsrelevante Aufgaben gerne ignorieren. Dem kann dadurch entgegengewirkt werden, dass die Aufgaben deutlich sichtbar in das Lehrkonzept eingebettet werden und eine zeitnahe Rückmeldung der Ergebnisse an die Studierenden, nötigenfalls eine Anpassung der Vorlesung, erfolgt. Eine gute Möglichkeit, elektronische Aufgaben in das Lehrkonzept zu integrieren, ist das Just-in-Time-Teaching. Dabei bearbeiten Studierende im Selbststudium bereits vor der Präsenzveranstaltung Aufgaben, die in elektronischer Form gestellt werden können. So kann der/ die Lehrende Einblick in die Ergebnisse nehmen und feststellen, wo Probleme liegen, die aufgegriffen werden müssen. Für die Studierenden schwierige 
Verfahren können nochmals erklärt, nicht oder falsch gelöste Aufgaben intensiv besprochen werden.

Studien haben gezeigt, dass zusätzlich gestellte Aufgaben, beispielsweise Hausaufgaben oder Zwischentests, grundsätzlich einen positiven Einfluss auf das Lernen der Studierenden haben (Case \& Swanson 2002: 9), da sie Lücken rechtzeitig erkennen und aufarbeiten können. Aufgrund des hohen Aufwands sind derartige zusätzliche Aufgaben und Tests in Papierform für die Lehrenden kaum zu bewältigen, in elektronischer Form aber möglich. Gleichzeitig ist hier aber Vorsicht geboten, da viele Studierende aus den Aufgaben auf die Anforderungen in dem Modul rückschließen, sodass die Diskrepanz im Anforderungsniveau zwischen den Übungs- und Klausuraufgaben nicht zu groß bzw. transparent sein sollte.

\section{E-ASSESSMENTS AN DER TH WILDAU [FH]: EIN PRAXISBEISPIEL}

Seit 2008 stellt die TH Wildau [FH] eine zentrale E-Learning-Plattform ${ }^{3}$ zur Verfügung (Open-Source-Software: Moodle), angesiedelt in der Hochschuleinheit „learn@wildau“. Diese wird zunehmend intensiv genutzt (im Sommersemester 2014 waren 3.567 Aktive auf der Plattform, die insgesamt 213 verschiedene Kurse genutzt haben). Sie enthält alle klassischen Funktionen einer E-Learning-Software und somit auch die Möglichkeit des Erstellens von elektronischen Tests. Deren Funktionalitäten sind bereits recht vielseitig, jedoch nicht so umfänglich, dass sie die Anforderungen an E-Assessments in allen Belangen erfüllen würden. Der Wunsch von Lehrenden, anspruchsvolle Tests und Prüfungen mit automatischer Korrekturfunktion durchzuführen, sodass u. a. auch Zwischentests ohne allzu viel zusätzlichen Aufwand möglich werden, mündete an der TH Wildau $[\mathrm{FH}]$ in den Erwerb von Lizenzen und den Aufbau der technischen Rahmenbedingungen für E-Assessments. Zusätzlich zur bestehenden Lernplattform steht nun die E-Assessment-Software „Questionmark ${ }^{\circledR}$ Perception“ zur Verfügung. Dieses Programm bietet wesentlich umfangrei- chere Gestaltungsmöglichkeiten und lässt außerdem einen Zugriff der Lizenzinhaber auf einen Fragenpool zu, wobei die Zugriffsmöglichkeiten für verschiedene Bereiche gestaffelt werden können (von „nicht sichtbar" bis „bearbeiten“). So können Fragen hochschulweit genutzt werden, was sich insbesondere für Selbsttestaufgaben in Grundlagenfächern anbietet. Die gemeinsame Nutzung eines Fragenpools hat zudem den Vorteil, dass mehrere Lehrende die Aufgaben fachlich-inhaltlich prüfen und die elektronische Funktion testen können.

Im vorhergehenden Abschnitt wurde gezeigt, dass es durchaus möglich ist, angemessene und niveauvolle Fragen elektronisch abzubilden und Studierende so zu prüfen. Das ermutigt dazu, E-Assessments in der Praxis zu testen, wobei der Schwerpunkt an der TH Wildau [FH] bisher eher auf diagnostischen und formativen Assessment-Formaten lag. Bis Mitte September 2014 wurden knapp 4.300 elektronische Tests absolviert.

Die folgenden Praxisbeispiele aus den Studiengängen Wirtschaftsinformatik (Modul E-Business, Bachelor, 5. Semester) und Business Management (Modul Wissensmanagement, Master, 3. Semester) stellten einen „Probelauf " für summative Tests, $d$. h. für Semesterabschlussklausuren, dar: Die Lehrveranstaltungen fanden im Wintersemester 2013/2014 statt. Die Gruppenstärke umfasste in einem Fall 20, im anderen 14 Studierende.

In beiden Lehrveranstaltungen erstellten die Studierenden jeweils eine schriftliche Arbeit bzw. eine Präsentation, die mit einer Note bewertet wurde. Ergänzend zu dieser Prüfungsleistung wurde in beiden Seminargruppen jeweils ein E-Assessment durchgeführt. Dieses musste lediglich "mit Erfolg“ absolviert werden, d.h. bei Erreichen von 50 \% der Punkte galt das gesamte Modul als „bestanden“ und der/die Studierende erhielt als Modulnote die Note der schriftlichen Arbeit bzw. Präsentation. Bei weniger als $50 \%$ der Punkte in der Klausur war das Modul „nicht bestanden" und das E-Assessment musste wiederholt werden.
Bei der Konzeption und Durchführung des E-Assessments standen in Anlehnung an Biella et al. (2010) die folgenden Aspekte im Vordergrund:

- Zeitaufwand zur Einarbeitung in die Software und Erstellung eines E-Assessments Nach Installation des Clients auf dem persönlichen Rechner hat die reine Einarbeitung in das Werkzeug im vorliegenden Fall ca. einen halben Arbeitstag gedauert, wobei dabei sicher nur ansatzweise die gesamte Funktionalität der Software erfasst werden konnte. Dennoch war es möglich, nach und nach die (bereits vorbereiteten Fragen) in verschiedene Fragetypen einzubetten und im Fragenpool abzuspeichern. Zusätzlich wurden pro Klausur noch einmal fünf Stunden benötigt, bis diese vollständig und korrekt eingegeben war. Beide Klausuren wurden so konzipiert, dass $50 \%$ der erreichbaren Punkte durch automatisch auswertbare Fragen erzielt werden konnten. Die übrigen Punkte konnten durch Freitextfragen erreicht werden, die von der Lehrenden separat bewertet werden mussten.

- Benötigte technische Infrastruktur sowie Personalbedarf Neben der Hard- und Software (Server und Client) ist es erforderlich, dass speziell ausgebildetes Personal zur Verfügung steht, um das System zu warten und die Lehrenden bei der Durchführung von E-Assessments zu unterstützen. Ansonsten benötigt man für die Durchführung lediglich ein Computerlabor, das für die Dauer der Klausur keinen Zugriff auf das Internet bzw. externe Informationsquellen zulässt. Die Studierenden erhalten einen individuellen Zugriff auf das System und haben keine andere Anwendung zur Auswahl als das jeweilige E-Assessment. Es sollte darauf geachtet werden, möglichst geräuscharme Tastaturen zu verwenden.

- Organisatorische Rahmenbedingungen Es hat sich als hilfreich und nützlich erwiesen, dass ein Administrator für die E-Assessment-

3 Erreichbar unter http://elearning.th-wildau.de. 
Software sowohl beim Verteilen der Zugangscodes als auch während der Bearbeitung und des Abschlusses der Klausur zugegen ist. Auch im Vorfeld hat dieser die von der Lehrenden erstellten Aufgaben hinsichtlich Aufbau und Fehlerfreiheit der Auswertung geprüft. Dieses Vier-Augen-Prinzip (siehe Abbildung 3) hat sich bei der Durchführung ausgezahlt, was sich $u$. a. daran gezeigt hat, dass es im Verlauf der Klausur keine Probleme bzw. Verständnisfragen gab.

\section{AUSBLICK}

E-Assessments sind - ebenso wie andere Anwendungen aus dem E-Learning - kein Allheilmittel und auch kein Selbstläufer. Sie bieten jedoch Vorteile für Lehrende und Studierende, wenn sie fach- und sachgerecht umgesetzt werden und eine hohe Passung zur Lehrveranstaltung aufweisen. Für Lehrende bieten E-Assessments eine Entlastung von Korrekturarbeiten, was semesterbegleitende Tests, die den Lernstand aktuell erfassen, ermöglicht. Die Fehlerdiagnose wird damit erleichtert, ohne dass dafür ein großer Anteil der Präsenzzeiten verwendet werden muss. Dabei muss es nicht immer um eine komplett automatische Korrektur gehen, bereits eine reduzierte Korrekturzeit ist als Vorteil zu werten, wobei eine manuelle Nachkorrektur in jedem Fall möglich sein sollte. Für die Studierenden bieten EAssessments in Form von Selbsttests ein Feedback zum eigenen Lernfortschritt, welches sie zeit- und ortsunabhängig erhalten können. Da Online-Tests den Mediengewohnheiten vieler Studierender entsprechen, kann die Motivation zum rechtzeitigen Aufarbeiten von Defiziten somit steigen (Bücking 2011: 41). Bei der Evaluation des Praxisbeispiels bewerteten die Studierenden die automatische Teilauswertung unmittelbar nach Beendigung der Klausur sehr positiv, da die meisten dadurch bereits abschätzen konnten, ob sie die Klausur bestanden hatten.

Die Qualität von Übungs- und Prüfungsaufgaben bzw. ihre Zusammenstellung in Tests ist von der Form - elektronisch oder schriftlich - zunächst unabhängig. Die grundsätzlichen Anforderungen sind in beiden Fällen gleich. Auch einige Einschrän- kungen treffen beide Formen der Leistungsüberprüfung, z. B. kann Präsentationskompetenz kaum anhand einer Klausur geprüft werden. Wird ein gemeinsamer Fragenpool genutzt, ist die Qualitätssicherung bei E-Assessments eher gewährleistet als bei klassischen schriftlichen Prüfungen, da alle zugreifenden Lehrenden die Aufgaben kontrollieren können. Aufgrund der erforderlichen Passung zwischen Lehrveranstaltung und E-Assessment wird allerdings häufig eine Anpassung der elektronischen Aufgaben aus dem Fragenpool auf den Studiengang, die Studiengruppe oder die Schwerpunktsetzungen des/der Lehrenden erfolgen müssen. Auch die Umwandlung in elektronische Aufgaben erfordert unter Umständen eine Anpassung oder Überarbeitung der von den Lehrenden verwendeten Aufgaben.

In diesem Beitrag wurde weiterhin gezeigt, dass die Erstellung kompetenzorientierter Fragen auch im Rahmen eines E-Assessments möglich ist, auch wenn sich dessen Anwendung nicht in allen Fällen anbietet. Beispielsweise ist bisher eine Bewertung des Lösungsweges nur bedingt, durch die Abfrage von Zwischenergebnissen, möglich. Zielen Aufgaben also nicht nur auf Ergebnisse, wird man weiterhin auf schriftliche Tests zurückgreifen oder beide Testarten kombiniert nutzen.

Ein weiterer wichtiger Aspekt ist der Auf- und Ausbau einer zuverlässigen technischen Hintergrundstruktur. Einführende Schulungen und ein laufender Support sind dabei notwendig, da ein flexibles Programm entsprechend umfangreiche Funktionalitäten hat sowie unter anderem Updates installiert werden müssen und TeilnahmeAccounts angelegt werden müssen. Bei der Durchführung elektronischer Prüfungen ist ein technischer Support vor Ort unabdingbar, damit bei Problemen sofort reagiert werden kann. Insbesondere wenn Abschlussklausuren als E-Assessments durchgeführt werden, sind die Anforderungen an die technische Sicherheit und die Dokumentation zur Wahrung von Datensicherheit und Datenschutz hoch, sodass entsprechende Rahmenbedingungen und Unterstützungsstrukturen notwendig sind. Diese liegen derzeit an der $\mathrm{TH}$ Wildau [FH] noch nicht komplett vor, sodass an dieser
Stelle noch Arbeiten notwendig sind. Das Praxisbeispiel hat jedoch zum Ausdruck gebracht, dass Studierende hinsichtlich E-Assessments wenig bis keine Berührungsängste haben und die Situation bei guter Organisation und Unterweisung problemlos bewältigen.

In diesem Sinne heißt es fortan, die Potenziale von E-Assessments weiter zu nutzen, deren Grenzen allerdings stets im Blick zu haben und die verschiedenen Testmöglichkeiten bzw. Instrumente zur Wissens- und Kompetenzüberprüfung intelligent $z u$ kombinieren. Hinsichtlich Technik und Organisation liegen an der TH Wildau [FH] bereits recht gute Rahmenbedingungen vor, die jedoch hinsichtlich formaler Aspekte (z. B. rechtliche Gesichtspunkte, Archivierung) noch auf "sicherere Füße“ gestellt werden müssen. Dennoch soll dieser Artikel dazu ermutigen, auch bereits die Möglichkeiten nicht-prüfungsrelevanter E-Assessments auszuschöpfen und die daraus entspringenden Vorteile zu nutzen. 


\section{LITERATUR}

Biella D, Huth D, Striewe M, Kohnen M, Dreibholz T, Becke M (2010) Organisation und Implementierung PC-gestützter Prüfungen an der Universität DuisburgEssen. http://www.e-teaching.org. Accessed $10 \mathrm{Sep}$ 2014

Bücking J (2011) Lessons Learned. Erfahrungen aus 7 Jahren eAssessment. Hamb eLearning Mag 7:40-42

Case SM, Swanson DB (2002) Constructing Written Test Questions for the Basic and Clinical Sciences, 3rd edn. National Board of Medical Examiners, Philadelphia, PA

Ehlers J, Guetl C, Höntzsch S, Usener C, Gruttmann S (2013) Prüfen mit Computer und Internet. Didaktik, Methodik und Organisation von E-Assessment. http://I3t.eu. Accessed 25 Jul 2014

Handke J, Schäfer AM (2012) E-Learning, E-Teaching und E-Assessment in der Hochschullehre. Eine Anleitung. Oldenbourg, München. ISBN: 978-3-486-70800-4

Krebs R (2004) Anleitung zur Herstellung von MC-Fragen und MC-Prüfungen. Universität Bern, Medizinische Fakultät, Institut für Aus-, Weiter- und Fortbildung IAWF, Abt. für Ausbildungs- und Examensforschung AAE. https://www.elearning.uni-mainz.de/files/2014/03/ Anleitung-zur-Herstellung-von-MC-Fragen-und-MCPrüfungen-für-die-ärztliche-Ausbildung.pdf. Accessed 11 Sep 2014

Paris SG, Lipson MY, Wixson KK (1983) Becoming a strategic reader. Contemp Educ Psychol 8:293-316. doi: 10.1016/0361-476X(83)90018-8

Schulz A, Apostolopoulos N (2011) eExaminations Put To Test. Potenziale computergestützter Prüfungen. Hamb eLearning Mag 7:37-39

\section{AUTOREN}

Dr. Xenia Valeska Jeremias

Technische Hochschule Wildau

ServiceZentrum Lernen und Lehren [SeL $\left.{ }^{2}\right]$

xenia.jeremias@th-wildau.de

\section{Birgit Sellmer, M.A}

Technische Hochschule Wildau

ServiceZentrum Lernen und Lehren $\left[\mathrm{SeL}^{2}\right]$

birgit.sellmer@th-wildau.de

\section{Prof. Dr. Ulrike Tippe}

Technische Hochschule Wildau

Vizepräsidentin für Studium, Lehre und Qualität

ulrike.tippe@th-wildau.de 